\title{
OPTIMAL CONTROL POLICY \\ FOR A BROWNIAN INVENTORY SYSTEM WITH CONCAVE ORDERING COST
}

\author{
DACHENG YAO,* Chinese Academy of Sciences \\ XIULI CHAO, ${ }^{* *}$ University of Michigan \\ JINGCHEN WU, ${ }^{* * *}$ University of Michigan
}

\begin{abstract}
In this paper we consider an inventory system with increasing concave ordering cost and average cost optimization criterion. The demand process is modeled as a Brownian motion. Porteus (1971) studied a discrete-time version of this problem and under the strong condition that the demand distribution belongs to the class of densities that are finite convolutions of uniform and/or exponential densities (note that normal density does not belong to this class), an optimal control policy is a generalized $(s, S)$ policy consisting of a sequence of $\left(s_{i}, S_{i}\right)$. Using a lower bound approach, we show that an optimal control policy for the Brownian inventory model is determined by a single pair $(s, S)$.
\end{abstract}

Keywords: Brownian inventory system; $(s, S)$ policy; concave ordering cost

2010 Mathematics Subject Classification: Primary 90B05

Secondary 90B30

\section{Introduction}

In this paper we study a continuous-review stochastic inventory system with a piecewise linear and increasing concave ordering cost $c(z) \geq 0$ for placing an order of quantity $z \geq 0$. That is, there exist some $a_{i}, i=0,1, \ldots, n$, satisfying

$$
0=a_{0}<a_{1}<\cdots<a_{n-1}<a_{n}=\infty
$$

such that $c(z)=K \mathbf{1}_{\{z>0\}}+c_{1} z$ when $0 \leq z<a_{1}$ and, for $i=1, \ldots, n-1$,

$$
c(z)=c\left(a_{i}\right)+c_{i+1} \cdot\left(z-a_{i}\right), \quad a_{i} \leq z<a_{i+1},
$$

where $\mathbf{1}_{\{A\}}$ is the indicator function taking value 1 if statement $\mathrm{A}$ is true and 0 otherwise, $K>0$, and

$$
c_{1}>c_{2}>\cdots>c_{n}>0
$$

is a sequence of decreasing positive constants. Note that $c(z)$ is continuous at all but point $z=0$; see Figure 1. Other nondecreasing concave ordering cost functions that are continuous on $z>0$ can be approximated, arbitrarily closely, by such piecewise linear concave functions.

\footnotetext{
Received 1 November 2013; revision received 3 November 2014.

* Postal address: Academy of Mathematics and Systems Science, Chinese Academy of Sciences, Beijing, 100190, China. Email address: dachengyao@amss.ac.cn

** Postal address: Department of Industrial and Operations Engineering, University of Michigan, Ann Arbor, MI 48109-2117, USA. Email address: xchao@umich.edu

*** Current address: 500 9th Ave N, Seattle, WA 98109, USA. Email address: wjch@umich.edu
} 


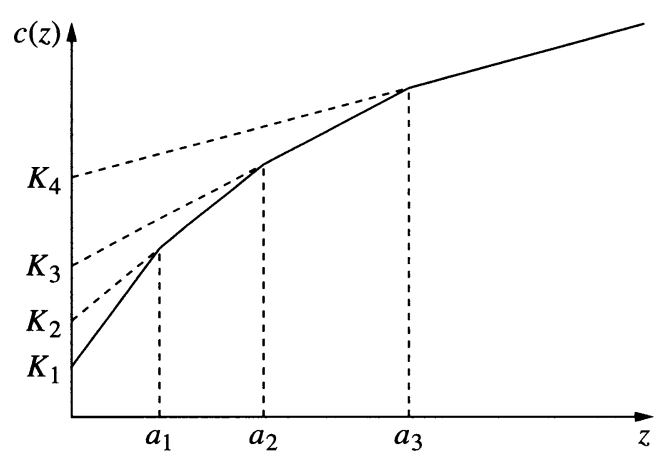

FIGURE 1: Ordering cost function $c(z)$.

The ordering cost described above includes many interesting special cases of practical interest. The following are two examples.

1. Classic inventory model with a setup cost plus proportional purchasing cost. This is obtained by setting $K>0$ and $n=1$, i.e. $a_{1}=\infty$. In this case, there is a setup cost $K$ for placing any positive order, and each unit costs $c_{1}$. This is one of the most studied stochastic inventory models in the literature; see, for example, Scarf [15].

2. Inventory models with incremental quantity discount. For example, if we set $a_{2}=\infty$ then we obtain a model that has incremental ordering quantity discount: the unit ordering cost is $c_{1}$ for an order quantity up to $a_{1}$, but any order quantity above $a_{1}$ has a discounted unit cost of $c_{2}<c_{1}$ (and $K$ is the order processing related cost). Ordering discount is commonly observed in practice; see, for example, Hax and Candea [9].

Due to its practical importance, increasing concave ordering cost has been studied in the literature. Porteus [12], [13] considered a periodic review inventory model with increasing concave ordering cost function. Under the strong condition that the demand distribution belongs to the class of densities that are finite convolutions of uniform and/or exponential densities, the author showed that there exists an optimal policy that is a generalized $(s, S)$ policy. It should be noted that normal density does not belong to this class.

In this paper we consider a stochastic inventory system where the demand is modeled by a Brownian motion process. As in the traditional inventory systems, in addition to an ordering cost, there are also holding and shortage costs, and the objective is to minimize the long-run average cost per unit time. We show that there is an optimal policy that is an $(s, S)$ policy. Our approach is based on first establishing a lower bound for the average cost within a large class of policies and then identifying an $(s, S)$ policy that achieves this lower bound thereby proving its optimality.

There are numerous papers in the literature on Brownian inventory systems with fixed plus proportional ordering costs. Bather [1] used Brownian motion to model the demand process and assumed that the inventory level could be adjusted by orders with a setup cost and proportional variable cost; and showed that an $(s, S)$ policy is optimal under a long-run average cost criterion. Richard [14] considered both infinite- and finite-horizon problems with a discounted cost criterion, and he presented sufficient conditions for the optimality of an $(d, D, U, u)$ policy among the class of impulse control policies. See also Constantinides and Richard [2]. Harrison and Taylor [7] studied a similar discounted cost optimal control problem 
of a Brownian model, and they imposed the condition that the state of the system is nonnegative and obtained an optimal impulse control policy for the case with or without a setup cost. Harrison et al. [8] proposed another Brownian model for a cash management problem in which the state of the system could be instantaneously increased or decreased, and the authors showed that a $(0, q, Q, S)$ policy is optimal for a discounted cost criterion. Sulem [16] investigated the issue of computing the control parameters of an optimal policy for a one-sided impulse control problem based on the work of [2]. Ormeci et al. [11] considered linear holding and shortage cost rate and extended the result of [8] to a long-run average-cost criterion. They also generalized the result to the case when there is a finite adjustment constraint in the impulse control policies. Dai and Yao [3], [4] further extended the model of [8] and [11] to convex holding and shortage costs and obtained an optimal control policy under either average or discounted cost criterion. $\mathrm{Wu}$ and Chao [17] studied a stochastic production system in which replenishment cannot be instantaneous, but instead must be gradual. They modeled both the production and demand processes as Brownian motions and characterized the structure of an optimal policy.

The rest of this paper is organized as follows. In the next section we present the mathematical formulation. We establish a lower bound for the average cost of an admissible policy in Section 3, analyse the average cost of the stochastic inventory system in Section 4, and prove the optimality of an $(s, S)$ policy in Section 5. For clarity the results are presented for the strict quasi-convex holding and shortage cost, and the general quasi-convex cost case is presented in Section 6.

\section{Model and result}

Let $\Omega$ be the space of all continuous functions $\omega:[0, \infty) \rightarrow \mathbb{R}$. Let $B=\{B(t): t \geq 0\}$ be a standard Brownian motion (with mean 0 and variance 1 ), and $\mathcal{F}=\left\{\mathscr{F}_{t}, t \geq 0\right\}$ be the natural filtration generated by $B$ with probability measure $\mathbb{P}$. Then, $(\Omega, \mathcal{F}, \mathbb{P})$ forms the probability space under consideration.

We consider an inventory system with its demand process modeled by a Brownian motion with drift parameter $\mu>0$ and variance parameter $\sigma$. That is, the total demand up to time $t$ is modeled by

$$
D(t)=\mu t+\sigma B(t), \quad t \geq 0 .
$$

The demand process above can be understood as the netput process, which captures the difference between the demand and some (nominal) input process such as customer returns. For convenience, we call this netput process the demand process.

The cost structure is described as follows. Let $Z(t)$ denote the inventory level of the system at time $t$. When the inventory level is $z$, it incurs a holding/penalty cost at rate $h(z)$. If $z \geq 0$ then $h(z)$ is the holding cost rate for having $z$ units on hand; while if $z<0$ then $h(z)$ is the penalty cost rate for having backlog level $-z>0$. To raise the inventory level, the firm can order from an outside supplier. The cost of ordering $z$ units of product, which is delivered immediately, is given by $c(z)$ as defined in (1).

A policy $\varphi$ is a sequence $\left\{\left(T_{n}, \xi_{n}\right), n \geq 0\right\}$, where $T_{0}=0, T_{n}$, the time of the $n$th replenishment, is a stopping time and $\xi_{n}$, the size of the $n$th replenishment, is $\mathcal{F}_{T_{n}}$ measurable. We shall focus on the following class of policies $\Psi$. For each policy $\varphi$ in $\Psi$, there exists some finite number $M_{\varphi}$ such that the policy $\varphi$ does not place an order when the inventory level is greater than $M_{\varphi}$. Because $M_{\varphi}$ can be arbitrarily large, $\Psi$ contains all policies of practical interest. In what follows, we say a policy $\varphi$ is admissible if $\varphi \in \Psi$. 
Suppose that the initial inventory level at time 0 is $x$, and assume that $Z(t)$ is right-continuous with left limits. Under an admissible policy $\varphi=\left\{\left(T_{n}, \xi_{n}\right), n \geq 0\right\}$, the inventory process $Z(t)$ is given by

$$
Z(t)=x-D(t)+\sum_{n=0}^{N(t)} \xi_{n}=x-\mu t-\sigma B(t)+\sum_{n=0}^{N(t)} \xi_{n},
$$

where $N(t)=\sup \left\{n: T_{n} \leq t\right\}$ is the number of inventory replenishments up to time $t$ (not including the possible replenishment at time 0 ). We assume that the policy satisfies

$$
\mathbb{E}_{x}[N(t)]<\infty, \quad \mathbb{E}_{x}\left[\sum_{n=0}^{N(t)} \xi_{n}\right]<\infty \quad \text { for each } t \geq 0,
$$

where $\mathbb{E}_{x}$ stands for the conditional expectation given $Z\left(0_{-}\right)=x$.

Given that the initial inventory level is $Z(0-)=x$, the long-run average cost for the system under an admissible policy $\varphi=\left\{\left(T_{n}, \xi_{n}\right), n \geq 0\right\}$ is defined as

$$
C_{\text {avg }}(x, \varphi)=\limsup _{T \rightarrow \infty} \frac{1}{T} \mathbb{E}_{x}\left[\int_{0}^{T} h(Z(t)) \mathrm{d} t+\sum_{n=0}^{N(T)} c\left(\xi_{n}\right)\right]
$$

The objective in this paper is to characterize an optimal admissible policy $\varphi^{*}$ that satisfies

$$
C_{\mathrm{avg}}\left(x, \varphi^{*}\right)=\min _{\varphi \in \Psi} C_{\mathrm{avg}}(x, \varphi)
$$

We assume that the holding and penalty cost function $h: \mathbb{R} \rightarrow[0, \infty)$ satisfies the following technical conditions.

Assumption 1. The cost function $h: \mathbb{R} \rightarrow \mathbb{R}_{+}$satisfies the following conditions:

(i) $h$ is continuous and quasi-convex;

(ii) $h$ has a minimum at $h(0)=0$;

(iii) $\lim _{|x| \rightarrow \infty} h(x)=+\infty$;

(iv) $h$ is polynomially bounded, i.e. there exist constants $A_{i}, i=1,2$ and an integer $m \in \mathbb{N}^{+}$ such that $h(x) \leq A_{1}+A_{2}|x|^{m}$ for all $x \in \mathbb{R}$.

(v) There exist scalars $p>0$ and $q$ such that $h(x) \geq-p x+q$.

Conditions (i)-(v) are standard assumptions in the inventory literature and are satisfied by most applications of practical interest. Condition (v) assumes that the cost rate function $h(x)$ is bounded below by a linear function, which holds, for example, if $h(x)$ is differentiable and $\lim \sup _{x \rightarrow-\infty} h^{\prime}(x)<0$.

To present the main result of this paper, we define $n$ positive (and increasing) values

$$
0<K_{1}<K_{2}<\cdots<K_{n}
$$

as follows: $K_{1}=K$ and, for $1<i<n$,

$$
K_{i+1}=K_{i}-\left(c_{i+1}-c_{i}\right) a_{i}
$$


See Figure 1 for an illustration of $K_{i}$. Moreover, let $\lambda=2 \mu / \sigma^{2}$ and define a function $G(x)$ by

$$
G(x)=\frac{2}{\sigma^{2}} \int_{x}^{\infty} h(\xi) \mathrm{e}^{-\lambda(\xi-x)} \mathrm{d} \xi
$$

Let $y^{*}$ be a minimizer of $G$. Furthermore, define a setup cost function $\mathcal{K}(Q)$ by $\mathcal{K}(0)=0$ and, for $1 \leq i \leq n$,

$$
\mathcal{K}(Q)=K_{i} \quad \text { if } a_{i-1}<Q \leq a_{i} .
$$

The following is the main result of this paper.

Theorem 1. An optimal control policy of the Brownian stochastic inventory system with concave ordering cost is an $(s, S)$ policy. In addition, the policy parameters $s$ and $S$ of this optimal policy satisfy

(i) $s \leq y^{*} \leq S$;

(ii) $G(s)=G(S)$;

(iii) $(S-s) G(S)-\int_{s}^{S} G(z) \mathrm{d} z=\mathcal{K}(S-s)$.

To prove that an optimal policy for the system under consideration assumes the desired form, we take the following approach. We first present a lower bound for the average cost function for an admissible policy within a large class of policies, and then identify a simple $(s, S)$ policy whose average cost achieves this lower bound. Finally, we show that if a policy does not belong to this class of policies then its average cost is equal to $\infty$; hence, it cannot be optimal. This implies that the identified $(s, S)$ policy is optimal.

\section{Lower bound}

In this section we establish a lower bound for the long-run average cost (2) for a policy within a large class of policies. To that end, we first state the generalized Itô formula; see, for example, Harrison [6, p. 71].

Lemma 1. Assume that $f$ is continuously differentiable and has a continuous second order derivative at all but a finite number of points. Then

$$
f(Z(T))=f(Z(0))+\int_{0}^{T} \Gamma f(Z(t)) \mathrm{d} t+\sigma \int_{0}^{T} f^{\prime}(Z(t)) \mathrm{d} B(t)+\sum_{n=1}^{N(T)} \theta_{n},
$$

where

$$
\Gamma f(x)=\frac{1}{2} \sigma^{2} f^{\prime \prime}(x)-\mu f^{\prime}(x) \text { for each } x \in \mathbb{R}
$$

such that $f^{\prime \prime}(x)$ exists, and $\theta_{n}=f\left(Z\left(T_{n}\right)\right)-f\left(Z\left(T_{n}-\right)\right)$.

The following theorem presents a lower bound for the optimal long-run average cost in (2) within a class of admissible policies.

Theorem 2. Suppose that $f$ satisfies the conditions in Lemma 1 and there is a constant $\gamma$ such that

(i) $\Gamma f(x)+h(x) \geq \gamma$ for each $x \in \mathbb{R}$ such that $f^{\prime \prime}(x)$ exists;

(ii) $f(x)-f(y) \geq-c(x-y)$ for $y<x$. 
If $\mathbb{E}_{x}\left[\int_{0}^{t}\left(f^{\prime}(Z(t))\right)^{2} \mathrm{~d} t\right]<\infty$ for each finite time $t$, then $C_{\mathrm{avg}}(x, \varphi) \geq \gamma$ for any initial state $x \in \mathbb{R}$ and any admissible policy $\varphi \in \Psi_{f}$, where

$$
\Psi_{f} \triangleq\left\{\varphi: \liminf _{T \rightarrow \infty} \frac{1}{T} \mathbb{E}_{x}[f(Z(T))] \leq 0 \text { for all } x \in \mathbb{R}\right\} .
$$

Proof. For an admissible policy $\varphi=\left\{\left(T_{n}, \xi_{n}\right): n \geq 0\right\}$, (4) implies that

$$
\begin{aligned}
f(Z(T)) & =f(Z(0))+\int_{0}^{T} \Gamma f(Z(t)) \mathrm{d} t+\sigma \int_{0}^{T} f^{\prime}(Z(t)) \mathrm{d} B(t)+\sum_{n=1}^{N(T)} \theta_{n} \\
& =f(x)+\int_{0}^{T} \Gamma f(Z(t)) \mathrm{d} t+\sigma \int_{0}^{T} f^{\prime}(Z(t)) \mathrm{d} B(t)+\sum_{n=0}^{N(T)} \theta_{n} \\
& \geq f(x)+\gamma T-\int_{0}^{T} h(Z(t)) \mathrm{d} t+\sigma \int_{0}^{T} f^{\prime}(Z(t)) \mathrm{d} B(t)-\sum_{n=0}^{N(T)} c\left(\xi_{n}\right),
\end{aligned}
$$

where the second equality follows from $\theta_{0}=f(Z(0))-f(Z(0-))=f(Z(0))-f(x)$, and the inequality is due to Theorems $2(\mathrm{i})$ and $2(\mathrm{ii})$.

If $\mathbb{E}_{x}\left[\int_{0}^{t}\left(f^{\prime}(Z(t))\right)^{2} \mathrm{~d} t\right]<\infty$ then $\int_{0}^{T} f^{\prime}(Z(t)) \mathrm{d} B(t)$ is a martingale. Taking the expectation on both sides of $(6)$ yields

$$
\mathbb{E}_{x}[f(Z(T))] \geq f(x)+\gamma T-\mathbb{E}_{x}\left[\int_{0}^{T} h(Z(t)) \mathrm{d} t+\sum_{n=0}^{N(T)} c\left(\xi_{n}\right)\right]
$$

Dividing both sides by $T$ and taking the limit superior as $T \rightarrow \infty$, we obtain

$$
C_{\text {avg }}(x, \varphi) \geq \gamma-\liminf _{T \rightarrow \infty} \frac{1}{T} \mathbb{E}_{x}[f(Z(T))] \geq \gamma .
$$

The last inequality follows from the assumption that $\varphi \in \Psi_{f}$.

\section{Analysis of the cost function}

We first analyse the cost function of the inventory system with concave ordering cost operating under a given $(s, S)$ policy. Under the said policy, whenever the inventory level drops to $s$, an order is placed to raise the inventory level to $S$. It is well known (see, for example, [1]) that an $(s, S)$ policy is optimal for the special cases of fixed plus proportional ordering costs.

The inventory process $Z(t)$ operating under an $(s, S)$ policy is a regenerative process if we define a cycle as follows. Without loss of generality, let us suppose that the inventory level starts from $s$, and an order of the quantity $S-s$ is placed. Then $Z(t)$ drops from $S$ with drift $-\mu$ and variance parameter $\sigma^{2}$ until it hits $s$, indicating the end of a cycle. The random duration of a cycle is the time it takes for the inventory level to drop from $S$ to $s$, which we denote by $\tau$. The total cost of a cycle is the summation of the ordering $\operatorname{cost} c(S-s)$ and the holding/penalty cost incurred while the inventory level drops from $S$ to $s$. Due to the regenerative structure, the long-run average system cost is equal to the expected cost during one cycle divided by the 
expected length of the cycle. See, for example, [10, Theorem 6-8, p. 184]. Thus, the long-run average cost under a given $(s, S)$ policy with $s<S$ is

$$
\gamma(s, S):=C_{\text {avg }}(s,(s, S))=\frac{c(S-s)+\mathbb{E}_{S}\left[\int_{0}^{\tau} h(Z(t)) \mathrm{d} t\right]}{\mathbb{E}_{S}[\tau]},
$$

where ':=' is 'defined as', $c(S-s)$ is the ordering cost in one cycle, $\mathbb{E}_{S}\left[\int_{0}^{\tau} h(Z(t)) \mathrm{d} t\right]$ is the expected holding/shortage cost in one cycle given that the system starts with inventory level $S$ (after placing the order), and $\tau$ denotes the length of one cycle and satisfies $\mu \tau+\sigma B_{\tau}=S-s$.

First, it follows from $\mu \tau+\sigma B_{\tau}=S-s$ that $\mathbb{E}_{S}[\tau]=(S-s) / \mu$. Secondly, the behavior of $Z(t)$ during the cycle allows us to write $\mathbb{E}_{S}\left[\int_{0}^{\tau} h(Z(t)) \mathrm{d} t\right]=\int_{s}^{S} G(z) \mathrm{d} z$, where $G(x)$ is defined in (3); see [17, Equation (13)]. Therefore, we have

$$
\gamma(s, S)=\frac{\mu\left(c(S-s)+\int_{s}^{S} G(z) \mathrm{d} z\right)}{S-s} .
$$

Based on Assumption 1, we have the following result.

Lemma 2. It holds that $G(x)$ is differentiable, quasi-convex, and $\lim _{|x| \rightarrow \infty} G(x)=\infty$.

Proof. By substituting $y=x-\xi$, from (3), we obtain

$$
G(x)=\frac{1}{\mu} \int_{-\infty}^{0} \lambda \mathrm{e}^{\lambda y} h(x-y) \mathrm{d} y=\frac{1}{\mu} \mathbb{E}[h(x-Y)],
$$

where the random variable $Y$ has density function $\rho(y)=\lambda \mathrm{e}^{\lambda y}$, where $y \leq 0$. Since $\ln \rho(y)=$ $\ln \lambda+\lambda y$, the density function $\rho(y)$ is a log-concave function. By the assumption that $h$ is a quasi-convex function, we conclude that $G(x)$ is a quasi-convex function as well; see [5, Theorem 1.10]. The function $G$ can also be written as

$$
G(x)=\frac{\lambda}{\mu} \mathrm{e}^{\lambda x} \int_{x}^{\infty} h(\xi) \mathrm{e}^{-\lambda \xi} \mathrm{d} \xi .
$$

This function is the product of two differentiable functions; hence, it is also differentiable. The fact that $\lim _{x \rightarrow \pm \infty} G(x)=\infty$ follows from the assumption that $\lim _{x \rightarrow \pm \infty} h(x)=\infty$.

By letting $Q=S-s \geq 0$ be the ordering quantity, we can write the average cost function $\gamma(s, S)$ in $(8)$ as

$$
\gamma(s, Q)=\frac{\mu\left(c(Q)+\int_{s}^{s+Q} G(z) \mathrm{d} z\right)}{Q} .
$$

For ease of exposition, in this section we assume that $G(x)$ is strictly quasi-convex, i.e. it strictly decreases before reaching a minimum at its unique minimizer $y^{*}$ and then strictly increases. Our results are extended to the case of weakly quasi-convex $G(x)$ in Section 6.

To minimize $\gamma(s, Q)$, in the following we first solve $\min _{s} \gamma(s, Q)$ for fixed $Q$, and then optimize the resulting objective function over $Q \geq 0$. It follows from

$$
\frac{\partial \gamma(s, Q)}{\partial s}=\mu \frac{G(s+Q)-G(s)}{Q}
$$

and Lemma 2 that $\gamma(s, Q)$ is quasi-convex in $s$. Let $s(Q)$ be the minimizer of $\gamma(s, Q)$, then it follows from Lemma 2 that $s(Q)$ is well defined for any $Q \geq 0$. 
Note that when $Q$ is fixed, the optimization problem of $\gamma(s, Q)$ over $s$ is the same as that of the classic stochastic inventory control problem with fixed ordering cost; see, for example, [18, Lemma 2 and Lemma 3], for which the following results are known to hold.

Lemma 3. For a given $Q$, the minimizer of $s=s(Q)$ of $\gamma(s, Q)$ satisfies

(i) $s(Q)$ is decreasing in $Q$; and $s(Q)+Q$ is increasing in $Q$;

(ii) $G(s(Q))=G(s(Q)+Q)$;

(iii) $s(Q) \leq y^{*}$ and $s(Q)+Q \geq y^{*}$.

Remark 1. We note that under the assumption that $G(x)$ is strictly quasi-convex, $s(Q)$ and $s(Q)+Q$ are uniquely determined and are strictly decreasing and increasing, respectively, in $Q$. If $G(x)$ is weakly quasi-convex, then we can show that there exists at least one optimal $s(Q)$ and at least one minimizer $y^{*}$ of $G(x)$ that satisfy Lemmas 3(ii) and 3(iii), but Lemma 3(i) will be changed to $\left(\mathrm{i}^{\prime}\right), s(Q)$ is nonincreasing in $Q$, and $s(Q)+Q$ is nondecreasing in $Q$. This, however, does not affect our subsequent analysis.

For convenience, we define the following function:

$$
\Delta(Q)=Q G(s(Q)+Q)-\int_{s(Q)}^{s(Q)+Q} G(z) \mathrm{d} z .
$$

By Lemma 3(i)-(iii) and the quasi-convexity of $G(x)$, we have $\Delta(Q) \geq 0$ and $\Delta(Q)$ is a nondecreasing function of $Q$.

We next optimize $\gamma(s, Q)$ on the range $Q \in\left[a_{i-1}, a_{i}\right]$. The following lemma characterizes the optimal solution.

Lemma 4. On the range $Q \in\left[a_{i-1}, a_{i}\right], i=1, \ldots, n-1$, the optimal $Q_{i}$ that minimizes $\gamma(s(Q), Q)$ is

(i) $Q_{i}=a_{i-1}$ if $\Delta\left(a_{i-1}\right)-K_{i}>0$;

(ii) $Q_{i}=a_{i}$ if $\Delta\left(a_{i}\right)-K_{i}<0$;

(iii) otherwise, $Q_{i} \in\left[a_{i-1}, a_{i}\right]$ and $\Delta\left(Q_{i}\right)=K_{i}$.

Proof. For any $i$ satisfying $1 \leq i \leq n-1$ and $z \in\left[a_{i-1}, a_{i}\right]$, the ordering cost function can be written as

$$
c(z)=K_{i}+c_{i} z
$$

(Note that at the boundary points of the interval $\left[a_{i-1}, a_{i}\right], c(z)$ has other representations.) Thus, the average cost function on this range can be written as

$$
\gamma(s, Q)=c_{i} \mu+\frac{\mu\left(K_{i}+\int_{s}^{s+Q} G(z) \mathrm{d} z\right)}{Q} .
$$

Since

$$
\min _{Q \geq 0, s} \gamma(s, Q)=\min _{Q \geq 0} \gamma(s(Q), Q),
$$

we take the derivative of $\gamma(s(Q), Q)$ with respect to $Q$ and apply the envelope theorem to obtain, when $Q \in\left[a_{i-1}, a_{i}\right]$,

$$
\frac{\mathrm{d} \gamma}{\mathrm{d} Q}(s(Q), Q)=\frac{\mu}{Q^{2}}\left(\Delta(Q)-K_{i}\right)
$$


This shows that, whether or not $\gamma(s(Q), Q)$ increases with respect to $Q$ depends on the sign of $\Delta(Q)-K_{i}$. Because $\Delta(Q)$ is nondecreasing in $Q$, it follows that $\gamma(s(Q), Q)$ is increasing on $\left[a_{i-1}, a_{i}\right]$ if $\Delta\left(a_{i-1}\right)-K_{i}>0$; hence, by the continuity of $\gamma(s(Q), Q)$ in $Q$, it implies that $Q_{i}=a_{i-1}$ if $\Delta\left(a_{i-1}\right)-K_{i}>0$. Similar argument shows that $Q_{i}=a_{i}$ if $\Delta\left(a_{i}\right)-K_{i}<0$.

Since $\Delta(Q)$ is nondecreasing in $Q, \Delta(Q)-K_{i}$ cannot cross from negative to positive multiple times, and by (10), $\mathrm{d} \gamma(s(Q), Q) / \mathrm{d} Q$ cannot cross from negative to positive multiple times either. This shows that $\gamma(s(Q), Q)$ is quasi-convex on $\left[a_{i-1}, a_{i}\right]$. If neither Lemma 4(i) nor (ii) is satisfied, then $\gamma(s(Q), Q)$ is strictly decreasing at $a_{i-1}$ and strictly increasing at $a_{i}$. Thus, there must exist a $Q_{i} \in\left[a_{i-1}, a_{i}\right]$ such that $\Delta\left(Q_{i}\right)=K_{i}$.

Remark 2. For $i=n$, i.e. $Q \in\left[a_{n-1}, \infty\right)$, Lemma 4(ii) does not occur, and the optimal $Q_{n}$ satisfies either Lemma 4(i) or (iii). This is because, when $Q \rightarrow \infty, \lim _{Q \rightarrow \infty} \Delta(Q)-K_{n}=\infty$, so Lemma 4(ii) will never happen. Similarly, when $i=1, \Delta\left(a_{0}\right)-K_{1}=-K_{1}<0$; thus, Lemma 4(i) never happens for $i=1$, and $Q_{1}$ satisfies either Lemma 4(ii) or (iii).

Remark 3. The argument above uses the fact that $\Delta(Q)-K_{i}$ in (10) is nondecreasing to prove $\gamma(s(Q), Q)$ is quasi-convex in $Q$ on each interval $\left[a_{i-1}, a_{i}\right]$. However, it is not guaranteed that the derivative of $\gamma(s(Q), Q)$ with respect to $Q$, namely $\left(\mu / Q^{2}\right)\left(\Delta(Q)-K_{i}\right)$, is also nondecreasing; thus, we have no conclusion about the convexity of $\gamma$. Furthermore, even though $\gamma(s(Q), Q)$ is quasi-convex on each interval $\left[a_{i-1}, a_{i}\right]$, it is not quasi-convex on $Q \in \mathbb{R}^{+}$.

Using $\mathcal{K}(Q)$, we can write the derivative of $\gamma(s(Q), Q)$ on all $Q \geq 0$ and $Q \neq a_{i}(i=$ $1,2, \ldots, n)$ as

$$
\frac{\mathrm{d} \gamma}{\mathrm{d} Q}(s(Q), Q)=\frac{\mu}{Q^{2}}(\Delta(Q)-\mathcal{K}(Q)) .
$$

The function $\Delta(Q)-\mathcal{K}(Q)$ is continuous at all points except $a_{i}, i=0,1, \ldots, n$, and it has a negative jump of size $-\left(K_{i+1}-K_{i}\right)$ at $Q=a_{i}$. Thus, (11) holds for all $Q \geq 0$ if it is understood as the right and left derivatives, respectively, at $Q=a_{i}+$ and $Q=a_{i}-$. See Figure 2 for an illustration of $\Delta(Q)-\mathcal{K}(Q)$. Since $\Delta(Q)-\mathcal{K}(Q)$ is a piecewise nondecreasing function with downward jumps at $Q=a_{i},(i=1, \ldots, n)$, and $\Delta(0)-\mathcal{K}(0)=-K_{1}<0$, $\lim _{Q \rightarrow \infty}[\Delta(Q)-\mathcal{K}(Q)]=\infty$, there must exist at least one $Q>0$ with $\Delta(Q)-\mathcal{K}(Q)=0$. There may be multiple zero points for the function $\Delta(Q)-\mathcal{K}(Q)$, and there are usually multiple local minimum points for $\gamma(s(Q), Q)$.

The next result characterizes the values of an optimal $(s, S)$ that minimizes $\gamma(s, S)$. Note that it differs from Theorem 1 in that in Theorem 3 the optimization is over the class of $(s, S)$ policies and we search for the two parameters $s$ and $S$, while in Theorem 1 we search for an optimal policy among all admissible policies, including the subset of $(s, S)$ policies.

Theorem 3. An optimal $\left(s^{*}, S^{*}\right)$ that minimizes $\gamma(s, S)$ of $(8)$ satisfies all the conditions of Theorem 1. That is, $s^{*}$ and $S^{*}$ satisfy $s^{*} \leq y^{*} \leq S^{*}, G\left(s^{*}\right)=G\left(S^{*}\right)$, and Theorem 1(iii).

Proof. We first prove that, under the conditions in Lemma 4(i) and 4(ii), the minimizer of $\gamma(s(Q), Q)$ on $Q \geq 0$ cannot fall into the interval $\left[a_{i-1}, a_{i}\right]$.

For each $i$ we optimize $\gamma(s(Q), Q)$ on the interval $Q \in\left[a_{i-1}, a_{i}\right]$. If Lemma 4(iii) is satisfied then the optimizer $Q_{i}$ on the interval $\left[a_{i-1}, a_{i}\right]$ satisfies $\Delta\left(Q_{i}\right)=K_{i}$. Otherwise, if $\Delta\left(a_{i-1}\right)-K_{i}>0$ (note that by Remark 2, this does not occur for $i=1$ ) then the optimal solution of $\gamma(s(Q), Q)$ is $Q=a_{i-1}$ and it also implies that $\Delta\left(a_{i-1}\right)-K_{i-1}>0$. The latter further implies that $\gamma(s(Q), Q)$ is strictly increasing at $Q=a_{i-1}$; hence, the minimizer of $\gamma(s(Q), Q)$ on $\left[a_{i-2}, a_{i-1}\right]$ is less than $a_{i-1}$ and the optimal solution of $\gamma(s(Q), Q)$ on 


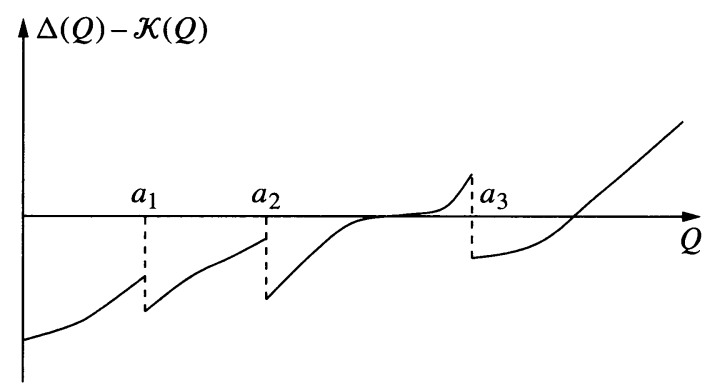

Figure 2: The graph of $\Delta(Q)-\mathcal{K}(Q)$.

$\left[a_{i-2}, a_{i}\right]$ is less than $a_{i-1}$. This shows that the minimizer of $\gamma(s(Q), Q)$ on the entire $Q \geq 0$ cannot be in the interval $\left[a_{i-1}, a_{i}\right]$. Similarly, if $\Delta\left(a_{i}\right)-K_{i}<0$ then the optimal solution of $\gamma(s(Q), Q)$ on $\left[a_{i-1}, a_{i}\right]$ is $Q=a_{i}$ and we have $\Delta\left(a_{i}\right)-K_{i+1}<0$, and the latter implies that the minimizer of $\gamma(s(Q), Q)$ on $\left[a_{i-1}, a_{i+1}\right]$ is greater than $a_{i}$ and the optimal solution of $\gamma(s(Q), Q)$ on $\left[a_{i-1}, a_{i+1}\right]$ is greater than $a_{i}$, implying that the minimizer of $\gamma(s(Q), Q)$ on $Q \geq 0$ cannot be in the interval $\left[a_{i-1}, a_{i}\right]$ either.

Because the minimizer of $\gamma(s(Q), Q)$ on $Q \geq 0$ exists and has to be in one of the intervals $\left[a_{i-1}, a_{i}\right], 1 \leq i \leq n$, the argument above shows that the optimal solution $Q^{*}$ is one of those $Q_{i}$ s that satisfy

$$
\Delta(Q)-\mathcal{K}(Q)=0 .
$$

Letting $s^{*}=s\left(Q^{*}\right)$ and $S^{*}=s\left(Q^{*}\right)+Q^{*}$, and applying Lemma 3 and (9), we complete the proof of Theorem 3 .

\section{Optimal policy}

Some preparations are needed to prove our main result. First, we introduce some notation. Let $\left(s_{i}, S_{i}\right)$ be the control parameters that minimize

$$
\gamma_{i}(s, S)=c_{i} \mu+\frac{\mu\left(K_{i}+\int_{s}^{S} G(z) \mathrm{d} z\right)}{S-s}
$$

under the constraint $S_{i}-s_{i} \in\left[a_{i-1}, a_{i}\right]$, and let $Q_{i}=S_{i}-s_{i}$. By Lemma 4(i)-(iii) and $K_{1}<K_{2}<\cdots<K_{n}$, we have

$$
Q_{1}<Q_{2}<\cdots<Q_{n}
$$

Also, on account of the decreasing property of $s(Q)$ and the increasing property of $s(Q)+Q$ as proved in Lemma 3, it holds that

$$
s_{n}<s_{n-1}<\cdots<s_{1}<y^{*}<S_{1}<S_{2}<\cdots<S_{n} .
$$

Let $\bar{\gamma}_{i}$ be the corresponding minimum objective function value, i.e.

$$
\bar{\gamma}_{i}=\min _{Q \in\left[a_{i-1}, a_{i}\right]} \gamma(s(Q), Q)
$$

Moreover, denote

$$
\bar{\gamma}=\min _{i \in\{1,2, \ldots, n\}}\left\{\bar{\gamma}_{i}\right\}=\bar{\gamma}_{i}
$$


and

$$
s^{*}=s\left(Q_{i^{*}}\right), \quad S^{*}=s\left(Q_{i^{*}}\right)+Q_{i^{*}} .
$$

In the rest of this section, we prove that the $\left(s^{*}, S^{*}\right)$ defined in (13) is an optimal policy for our original problem among the class of policies $\Psi$ of Section 2 . This is done in two steps. First, we define a value function $v$ and prove that the $\left(s^{*}, S^{*}\right)$ policy is optimal among all policies of $\Psi$ that are in $\Psi_{v}$ (see (5)). Secondly, we prove that if a policy of $\Psi$ is not in $\Psi_{v}$, then its average cost is equal to $\infty$; hence, it is worse than policy $\left(s^{*}, S^{*}\right)$.

Define a value function $v$ by

$$
v(x)= \begin{cases}\int_{s_{n}}^{x}\left(-\frac{\bar{\gamma}}{\mu}+G(y)\right) \mathrm{d} y & \text { for } x \geq s_{n}, \\ \left(-\frac{\bar{\gamma}}{\mu}+G\left(s_{n}\right)\right)\left(x-s_{n}\right) & \text { for } x<s_{n} .\end{cases}
$$

For convenience, we let

$$
\bar{G}(x)= \begin{cases}G(x) & \text { for } x \geq s_{n} \\ G\left(s_{n}\right) & \text { for } x<s_{n}\end{cases}
$$

Then, for $x \in \mathbb{R}$, we have

$$
v^{\prime}(x)=-\frac{\bar{\gamma}}{\mu}+\bar{G}(x)
$$

We now prove that the policy $\left(s^{*}, S^{*}\right)$ given by (13) is optimal among all policies in $\Psi_{v}$. By Theorem 2, it suffices to show that $v$ and $\bar{\gamma}$ defined in (12) satisfy Theorem 2(i) and (ii), which we verify next.

First, from (14) it is easy to see that $v^{\prime}$ is continuous at $x=s_{n}$; hence, $v^{\prime}$ is continuous in $x \in \mathbb{R}$, and that $v^{\prime \prime}(x)$ is continuous except at point $x=s_{n}$.

Next, we verify Theorem 2(i). Note that $G$ defined by (3) satisfies

$$
\frac{\sigma^{2}}{2} G^{\prime}(x)-\mu G(x)+h(x)=0 .
$$

To check Theorem 2(i), we apply (15) to obtain, for $x \geq s_{n}$,

$$
\Gamma v(x)+h(x)=\frac{\sigma^{2}}{2} G^{\prime}(x)-\mu\left(-\frac{\bar{\gamma}}{\mu}+G(x)\right)+h(x)=\bar{\gamma} .
$$

For $x<s_{n}$, we have

$$
\Gamma v(x)+h(x)=\bar{\gamma}-\mu G\left(s_{n}\right)+h(x)=\bar{\gamma}+\mu\left(G(x)-G\left(s_{n}\right)\right)-\frac{\sigma^{2}}{2} G^{\prime}(x) \geq \bar{\gamma},
$$

where the second equality follows from (15), and the inequality follows from the fact that $G$ is quasi-convex and that $x<s_{n} \leq y^{*}$.

To verify Theorem 2(ii), we first note that for any $i \in\{1, \ldots, n\}$ and $x-y \in\left[a_{i-1}, a_{i}\right]$, if $y<s_{n}$ then we have

$$
\begin{aligned}
\frac{\mu\left(K_{i}+\int_{y}^{x} \bar{G}(z) \mathrm{d} z\right)}{x-y} & \geq \frac{\mu\left(K_{i}+\int_{s_{n}}^{\left(s_{n}+(x-y)\right) \wedge S_{n}} \bar{G}(z) \mathrm{d} z\right)}{\left(s_{n}+(x-y)\right) \wedge S_{n}-s_{n}} \\
& =\frac{\mu\left(K_{i}+\int_{s_{n}}^{\left(s_{n}+(x-y)\right) \wedge S_{n}} G(z) \mathrm{d} z\right)}{\left(s_{n}+(x-y)\right) \wedge S_{n}-s_{n}}
\end{aligned}
$$


where $a \wedge b=\min \{a, b\}$ for any numbers $a$ and $b$. To see why this holds, we consider two cases separately: $i \in\{1, \ldots, n-1\}$ and $i=n$. First, for $i \in\{1, \ldots, n-1\}$, we have $x-y \leq a_{i} \leq a_{n-1} \leq S_{n}-s_{n}$; hence, $\left(s_{n}+(x-y)\right) \wedge S_{n}=s_{n}+(x-y)$ and $\bar{G}\left(x_{1}\right) \leq \bar{G}\left(x_{2}\right)$ for $x_{1} \in\left[s_{n}, S_{n}\right]$ and $x_{2} \notin\left[s_{n}, S_{n}\right]$. For an illustration, see Figure 3. Secondly, for $i=n$, if $S_{n}-s_{n} \geq x-y$, then the proof is similar to that when $i \in\{1, \ldots, n-1\}$. If $S_{n}-s_{n}<x-y$, then it follows from Remark 2 that $\Delta\left(S_{n}-s_{n}\right) \geq K_{n}$, i.e.

$$
\bar{G}\left(s_{n}\right)=G\left(s_{n}\right)=G\left(S_{n}\right) \geq \frac{\mu\left(K_{n}+\int_{s_{n}}^{S_{n}} G(z) \mathrm{d} z\right)}{S_{n}-s_{n}}
$$

Thus, we have

$$
\begin{aligned}
\frac{\mu\left(K_{n}+\int_{y}^{x} \bar{G}(z) \mathrm{d} z\right)}{x-y} & \geq \frac{\mu\left(K_{n}+\int_{s_{n}}^{S_{n}} \bar{G}(z) \mathrm{d} z+\bar{G}\left(s_{n}\right)\left((x-y)-\left(S_{n}-s_{n}\right)\right)\right)}{x-y} \\
& \geq \frac{\mu\left(K_{n}+\int_{s_{n}}^{S_{n}} \bar{G}(z) \mathrm{d} z\right)}{S_{n}-s_{n}}
\end{aligned}
$$

where the first inequality follows from $S_{n}-s_{n}<x-y$ and $\bar{G}\left(x_{1}\right) \leq \bar{G}\left(x_{2}\right)$ for $x_{1} \in\left[s_{n}, S_{n}\right]$ and $x_{2} \notin\left[s_{n}, S_{n}\right]$, and the second inequality follows from $\bar{G}\left(s_{n}\right) \geq \mu\left(K_{n}+\int_{s_{n}}^{S_{n}} G(z) \mathrm{d} z\right) /\left(S_{n}-s_{n}\right)$. This shows that (16) is indeed satisfied.

Therefore, for any $i \in\{1, \ldots, n\}$ and $x-y \in\left[a_{i-1}, a_{i}\right]$, we have

$$
\begin{aligned}
c_{i} \mu+\frac{\mu\left(K_{i}+\int_{y}^{x} \bar{G}(z) \mathrm{d} z\right)}{x-y} & \geq c_{i} \mu+\frac{\mu\left(K_{i}+\int_{s_{n}}^{\left(s_{n}+(x-y)\right) \wedge S_{n}} G(z) \mathrm{d} z\right)}{\left(s_{n}+(x-y)\right) \wedge S_{n}-s_{n}} \\
& \geq c_{i} \mu+\min _{x-y \in\left[a_{i-1}, a_{i}\right]} \frac{\mu\left(K_{i}+\int_{y}^{x} G(z) \mathrm{d} z\right)}{x-y} \\
& =\bar{\gamma}_{i},
\end{aligned}
$$

where the first inequality follows from that if $y<s_{n},(16)$ holds and $\left(s_{n}+(x-y)\right) \wedge S_{n}-s_{n} \in$ $\left[a_{i-1}, a_{i}\right]$ when $x-y \in\left[a_{i-1}, a_{i}\right], i \in\{1, \ldots, n\}$. For any $x \geq y$, there exists an $i \in\{1, \ldots, n\}$

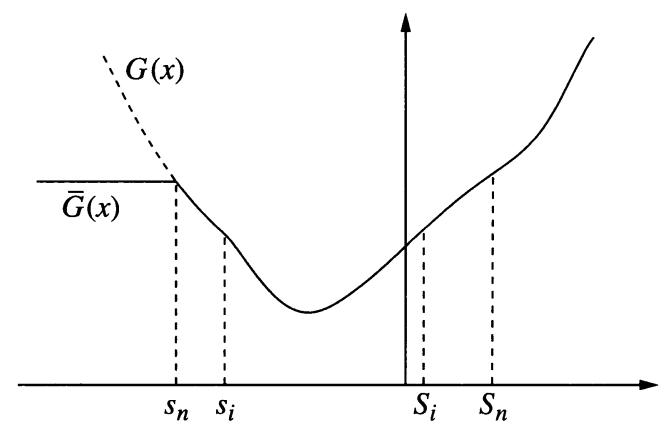

FIGURE 3: Relationship between $G(x)$ and $\bar{G}(x)$. 
such that $x-y \in\left[a_{i-1}, a_{i}\right]$, and $c(x-y)=K_{i}+c_{i}(x-y)$. We have

$$
\begin{aligned}
v(x)-v(y)+c(x-y) & =\int_{y}^{x}\left(v^{\prime}(z)+c_{i}\right) \mathrm{d} z+K_{i} \\
& =\int_{y}^{x}\left(-\frac{\bar{\gamma}}{\mu}+\bar{G}(z)+c_{i}\right) \mathrm{d} z+K_{i} \\
& \geq \int_{y}^{x}\left(-\frac{\overline{\gamma_{i}}}{\mu}+\bar{G}(z)+c_{i}\right) \mathrm{d} z+K_{i} \\
& =(x-y)\left(c_{i}+\frac{K_{i}+\int_{y}^{x} \bar{G}(z) \mathrm{d} z}{x-y}-\frac{\bar{\gamma}_{i}}{\mu}\right) \\
& \geq 0,
\end{aligned}
$$

where the second equality follows from (14), the first inequality follows from the definition of $\bar{\gamma}$, and the last inequality follows from (17). Thus, Theorem 2(ii) is proven to hold.

To complete the proof that policy $\left(s^{*}, S^{*}\right)$ is optimal among all policies in $\Psi_{v}$, by Theorem 2 we need to verify the condition that the inventory process of any policy $\varphi \in \Psi_{v}$ satisfies $\mathbb{E}_{x}\left[\int_{0}^{T}\left(v^{\prime}(Z(t))\right)^{2} \mathrm{~d} t\right]<\infty$. Note that $v^{\prime}(x)=-\bar{\gamma} / \mu+\bar{G}(x)$ and it can be easily shown that when $h(x)$ is polynomially bounded with degree $m$ then so is $\bar{G}(x)$. That is, there exist constants $a_{1}$ and $a_{2}$ such that $\left|v^{\prime}(x)\right| \leq a_{1}+a_{2}|x|^{m}$. For a policy $\varphi$ for which there exists an $M_{\varphi}$ such that it does not order whenever the inventory level is at or above $M_{\varphi}$, the inventory process is bounded between two processes: one is obtained by raising the inventory level to $M_{\varphi}$ whenever the inventory drops below it, and the other is to not order anything. Call these two inventory level processes $\bar{Z}(t)$ and $\underline{Z}(t)$, respectively. Then, for all $t \geq 0, \underline{Z}(t) \leq Z(t) \leq \bar{Z}(t)$. Hence,

$$
|Z(t)| \leq|\underline{Z}(t)|+|\bar{Z}(t)| .
$$

It is obvious that $\underline{Z}(t)$ is the Brownian motion process $x-\mu t+\sigma B(t)$, and $\bar{Z}(t)$ is the regulated Brownian motion process with a single barrier, which has a steady state distribution that is exponentially distributed; see [6, Sections 1.9 and 2.2] for the definition and analysis of regulated Brownian motion, and [6, Equation (1), Section 3.6] for its exponential steady state distribution. Applying these results, we obtain

$$
\begin{gathered}
\mathbb{P}(\underline{Z}(t) \leq y)=\Phi\left(\frac{-(y-x)-\mu t}{\sigma \sqrt{t}}\right), \quad y \in \mathbb{R}, \\
\mathbb{P}(\bar{Z}(t)>y)=\Phi\left(\frac{-y+\bar{Z}(0)-\mu t}{\sigma \sqrt{t}}\right)+\mathrm{e}^{-\lambda\left(y-M_{\varphi}\right)} \Phi\left(\frac{-y-\bar{Z}(0)+\mu t}{\sigma \sqrt{t}}\right), \quad y \geq M_{\varphi},
\end{gathered}
$$

where $\Phi(\cdot)$ is the standard normal distribution function; see $[6$, p. 49] for the distribution of $\bar{Z}(t)$. Then, we have $\mathbb{E}_{x}\left[\int_{0}^{T}|\underline{Z}(t)|^{2 m} \mathrm{~d} t\right]<\infty$ and $\mathbb{E}_{x}\left[\int_{0}^{T}|\bar{Z}(t)|^{2 m} \mathrm{~d} t\right]<\infty$. These results together with (18) and $\left|v^{\prime}(x)\right| \leq a_{1}+a_{2}|x|^{m}$ imply that $\mathbb{E}_{x}\left[\int_{0}^{T}\left(v^{\prime}(Z(t))\right)^{2} \mathrm{~d} t\right]<\infty$ for any $T \geq 0$.

We finally show that if a policy $\varphi \in \Psi$ is not in $\Psi_{v}$ then its average cost is equal to $\infty$; hence, it cannot be better than policy $\left(s^{*}, S^{*}\right)$. This concludes that policy $\left(s^{*}, S^{*}\right)$ is optimal among all policies in $\Psi$.

Consider a policy $\varphi \in \Psi \backslash \Psi_{v}$ with inventory process $\{Z(t) ; t \geq 0\}$. Because $\varphi \in \Psi$, there exists some large $M_{\varphi}$ such that $\varphi$ never orders when the inventory level is at or above $M_{\varphi}$. 
By assumption, we have $\liminf _{T \rightarrow \infty} \mathbb{E}_{x}[v(Z(T))] / T>0$; thus, there exist $T^{0}$ and $a>0$ such that for all $t>T^{0}, \mathbb{E}_{x}[v(Z(t))]>$ at. For an arbitrary $t \geq T^{0}$, we have

$$
\begin{aligned}
\mathbb{E}_{x}[v(Z(t))]= & \mathbb{E}_{x}\left[v(Z(t)) \mathbf{1}_{\left\{Z(t)>M_{\varphi}\right\}}\right]+\mathbb{E}_{x}\left[v(Z(t)) \mathbf{1}_{\left\{s_{n}<Z(t) \leq M_{\varphi}\right\}}\right] \\
& +\mathbb{E}_{x}\left[v(Z(t)) \mathbf{1}_{\left\{Z(t) \leq s_{n}\right\}}\right] \\
\leq & \mathbb{E}_{x}\left[v(Z(t)) \mathbf{1}_{\left\{Z(t)>M_{\varphi}\right\}}\right]+\sup _{s_{n}<Z \leq M_{\varphi}} v(z)+\mathbb{E}_{x}\left[v(Z(t)) \mathbf{1}_{\left\{Z(t) \leq s_{n}\right\}}\right]
\end{aligned}
$$

Clearly, $M(t)=Z(t) \mathbf{1}_{\left\{Z(t)>M_{\varphi}\right\}}$ represents the part of the process $Z(t)$ that is above $M_{\varphi}$. Recall that $Z(t) \leq \bar{Z}(t)$ for all $t \geq 0$ and $\bar{Z}$ is the regulated Brownian motion process with a single barrier $M_{\varphi}$, which has exponential steady state distribution. As $\lim _{x \rightarrow \infty} G(x)=\infty$ in Lemma 2 and $v^{\prime}(x)=-\bar{\gamma} / \mu+\bar{G}(x)$, when $M_{\varphi}$ is large enough we have $v^{\prime}(x)>0$ for $x>$ $M_{\varphi}$. Then, $\mathbb{E}_{x}\left[v(Z(t)) \mathbf{1}_{\left\{Z(t)>M_{\varphi}\right\}}\right] \leq \mathbb{E}_{x}\left[v(\bar{Z}(t)) \mathbf{1}_{\left\{\bar{Z}(t)>M_{\varphi}\right\}}\right]<\infty$, where the last inequality follows from the fact that $v$ is polynomially bounded. Let $a_{3}=\mathbb{E}_{x}\left[v(Z(t)) \mathbf{1}_{\{Z(t)>M \varphi\}}\right.$ and $a_{4}=\sup _{s_{n}<z \leq M_{\varphi}} v(z)$, then by $\mathbb{E}_{x}[v(Z(t))]>a t$, we obtain

$$
a t<a_{3}+a_{4}+\mathbb{E}_{x}\left[v(Z(t)) \mathbf{1}_{\left\{Z(t) \leq s_{n}\right\}}\right]
$$

On $Z(t) \leq s_{n}, v(Z(t))=-A Z(t)+B$. If $A \leq 0$ then $-A Z(t)+B \leq-A s_{n}+B$; hence,

$$
\mathbb{E}_{x}\left[v(Z(t)) \mathbf{1}_{\left\{Z(t) \leq s_{n}\right\}}\right] \leq-A s_{n}+B .
$$

It follows that, for large $t$,

$$
\mathbb{E}_{x}\left[v(Z(t)) \mathbf{1}_{\left\{Z(t) \leq s_{n}\right\}} \ngtr a t-a_{3}-a_{4} .\right.
$$

Thus, we must have $A>0$. We have

$$
\mathbb{E}_{x}\left[v(Z(t)) \mathbf{1}_{\left\{Z(t) \leq s_{n}\right\}}\right]=-A \mathbb{E}_{x}\left[Z(t) \mathbf{1}_{\left\{Z(t) \leq s_{n}\right\}}\right]+B \mathbb{P}\left\{Z(t) \leq s_{n}\right\}>a t-a_{3}-a_{4} .
$$

Consequently,

$$
\mathbb{E}_{x}\left[Z(t) \mathbf{1}_{\left\{Z(t) \leq s_{n}\right\}}\right]<-\frac{a t}{A}+\frac{a_{3}+a_{4}+B}{A} \leq-a_{5} t+a_{6},
$$

where $a_{5}$ and $a_{6}$ are some constants with $a_{5}>0$.

On the other hand, Assumption 1(v) implies that the existence of constant $p>0$ and $q$ such that $h(x) \geq-p x+q$ for all $x$. Hence, applying these results, we obtain

$$
\begin{aligned}
C_{\text {avg }}(x, \varphi) & \geq \limsup _{T \rightarrow \infty} \frac{1}{T} \int_{T^{0}}^{T} \mathbb{E}_{x}[h(Z(s))] \mathrm{d} s \\
& \geq \limsup _{T \rightarrow \infty} \frac{1}{T} \int_{T^{0}}^{T} \mathbb{E}_{x}\left[h(Z(s)) \mathbf{1}_{\left\{Z(s) \leq s_{n}\right\}}\right] \mathrm{d} s \\
& \geq \limsup _{T \rightarrow \infty} \frac{1}{T} \int_{T^{0}}^{T}\left(-p \mathbb{E}_{x}\left[Z(s) \mathbf{1}_{\left\{Z(s) \leq s_{n}\right\}}\right]+q \mathbb{P}\left\{Z(s) \leq s_{n}\right\}\right) \mathrm{d} s \\
& \geq \limsup _{T \rightarrow \infty} \frac{1}{T} \int_{T^{0}}^{T}\left(a_{7} s-a_{8}-q\right) \mathrm{d} s \\
& =\infty,
\end{aligned}
$$

where $a_{7}=a_{5} p, a_{8}=a_{6} p$. This shows that policy $\varphi$ cannot be better than policy $\left(s^{*}, S^{*}\right)$. 
To summarize, we have shown that among those policies of $\Psi$ that are in $\Psi_{v}$, an optimal policy is the $\left(s^{*}, S^{*}\right)$ identified, and any policy of $\Psi$ that is not in $\Psi_{v}$ has an average cost equal to $\infty$. It follows that the $\left(s^{*}, S^{*}\right)$ policy is optimal among all policies in $\Psi$, completing the proof of Theorem 1.

\section{General quasi-convex cost}

In this section we provide the technical details for the case when $G(\cdot)$ is weakly quasiconvex. Specifically, we give a rigorous definition for $s(Q)$ and prove its properties stated in Lemma 3. All other results follow the same lines of analyses as before.

For convenience, for a closed bounded interval $\Lambda$, we use $\min \Lambda$ and $\max \Lambda$ to denote the minimum and maximum points of $\Lambda$. Let $g=\min _{x} G(x)$ and $\Lambda_{0}=\arg \min _{x} G(x)$. For any $g>0$, we define

$$
\Lambda_{g}=\Lambda_{g, l} \cup \Lambda_{g, r},
$$

where $\Lambda_{g, l}=\left\{x<\min \Lambda_{0} ; G(x)=g+g\right\}, \Lambda_{g, r}=\left\{x>\max \Lambda_{0} ; G(x)=g+g\right\}$. Since $G(x)$ is continuous quasi-convex and $\lim _{x \rightarrow \pm \infty} G(x)=\infty, \Lambda_{g, l}$ and $\Lambda_{g, r}$ both exist and are bounded closed intervals. Similarly, we can show that for any $g^{\prime}>g$, if $x_{l}^{\prime} \in \Lambda_{g^{\prime}, l}$ and $x_{l} \in \Lambda_{g, l}$, then $x_{l}^{\prime}<x_{l}$; and if $x_{r}^{\prime} \in \Lambda_{g^{\prime}, r}$ and $x_{r} \in \Lambda_{g, r}$, then $x_{r}^{\prime}>x_{r}$.

Let $y^{*}=\left(\max \Lambda_{0}+\min \Lambda_{0}\right) / 2$. For $Q=0$, we define $g(Q)=0$; and for any $Q>0$, we define $g(Q)$ by

$$
\begin{aligned}
g(Q)= & \left\{g \geq 0: \text { there exists } x_{l}<y^{*}, x_{r}>y^{*},\right. \\
& \text { such that } \left.x_{r}-x_{l}=Q, g+\underline{g}=G\left(x_{l}\right)=G\left(x_{r}\right)\right\} .
\end{aligned}
$$

Since $G(x)$ is a continuous function, $g(Q)$ is never empty. Besides, we can show that $g(Q)$ is a single-valued function. If not, then we have $x_{l}, x_{l}^{\prime}<\min \Lambda_{0}, x_{r}, x_{r}^{\prime}>\max \Lambda_{0}$ with $x_{r}-x_{l}=Q, x_{r}^{\prime}-x_{l}^{\prime}=Q$, and $G\left(x_{l}\right)=G\left(x_{r}\right)=g+g<g^{\prime}+g=G\left(x_{l}^{\prime}\right)=G\left(x_{r}^{\prime}\right)$. By the quasi-convexity of $G(\cdot), x_{l}^{\prime}<x_{l}$ and $x_{r}^{\prime}>x_{r}$, which contradicts the condition that $x_{r}^{\prime}-x_{l}^{\prime}=x_{r}-x_{l}=Q$. Therefore, $g(Q)$ is a function and, according to the argument, $g(Q)$ is nondecreasing. We note that $g(Q)$ is not a one-to-one function, i.e. for $Q_{1} \neq Q_{2}$, it is possible that $g\left(Q_{1}\right)=g\left(Q_{2}\right)$. Finally, we show that $g(Q)$ is continuous, since if not, there is a $Q$ such that $\lim _{Q^{\prime} \rightarrow Q^{+}} g\left(Q^{\prime}\right)>\lim _{Q^{\prime} \rightarrow Q^{-}} g\left(Q^{\prime}\right)$. For any $g$ such that $\lim _{Q^{\prime} \rightarrow Q^{-}} g\left(Q^{\prime}\right)<g<$ $\lim _{Q^{\prime} \rightarrow Q^{+}} g\left(Q^{\prime}\right)$, since $\Lambda_{g, l}$ and $\Lambda_{g, r}$ are not empty, there exists $Q$ such that $g(Q)=g$, which is again a contradiction. Thus, we conclude that $g(Q)$ is continuous.

For $g>0$, we further define

$$
q_{g}=\min \left\{\Lambda_{g, r}\right\}-\max \left\{\Lambda_{g, l}\right\}, \quad Q_{g}=\max \left\{\Lambda_{g, r}\right\}-\min \left\{\Lambda_{g, l}\right\} .
$$

We note that if $\left|\Lambda_{g, l}\right|$ and $\left|\Lambda_{g, r}\right|\left(|\cdot|\right.$ is the length of the interval) are equal to 0 , then $q_{g}=Q_{g}$. Also, we define $s_{g}^{*}=\max \left\{x ; x \in \Lambda_{g, l}\right\}$ and $S_{g}^{*}=\min \left\{x ; x \in \Lambda_{g, r}\right\}$.

We define the function $s(Q)$ as

$$
s(Q)= \begin{cases}y^{*}-\frac{1}{2} Q & \text { if } Q \leq\left|\Lambda_{0}\right|, \\ s_{g(Q)}^{*} & \text { if } Q>\left|\Lambda_{0}\right| \text { and }\left|\Lambda_{g(Q), l}\right|=\left|\Lambda_{g(Q), r}\right|=0, \\ s_{g(Q)}^{*}-p\left(Q-q_{g(Q)}\right) & \text { otherwise, }\end{cases}
$$

where

$$
p=\frac{\left|\Lambda_{g, l}\right|}{\left|\Lambda_{g, l}\right|+\left|\Lambda_{g, r}\right|} .
$$


We now prove that $s(Q)$ is a nonincreasing function of $Q$. For any $Q_{1}<Q_{2}$, if $g\left(Q_{1}\right)=$ $g\left(Q_{2}\right)$, according to the definition of $s(Q)$, then $s\left(Q_{2}\right) \leq s\left(Q_{1}\right)$. Otherwise, $g\left(Q_{2}\right)>g\left(Q_{1}\right)$ then $\Lambda_{g\left(Q_{2}\right), l}$ is to the left of $\Lambda_{g\left(Q_{1}\right), l}$ as discussed before; thus, $s\left(Q_{2}\right)<s\left(Q_{1}\right)$. Next, we show that $s(Q)$ is continuous. Since $s(Q)$ has been shown to be nonincreasing, if $s(Q)$ is not continuous then there exists $Q$ such that $\lim _{Q^{\prime} \rightarrow Q^{-}} s\left(Q^{\prime}\right)>\lim _{Q^{\prime} \rightarrow Q^{+}} s\left(Q^{\prime}\right)$. For any $s^{\prime} \in\left(\lim _{Q^{\prime} \rightarrow Q^{-}} s\left(Q^{\prime}\right), \lim _{Q^{\prime} \rightarrow Q^{+}} s\left(Q^{\prime}\right)\right)$, let $g=G\left(s^{\prime}\right)-g$. If $\left|\Lambda_{g, l}\right|=\left|\Lambda_{g, r}\right|=0$, then $Q=S_{g}^{*}-s_{g}^{*}$ makes $s(Q)=s^{\prime}$. Otherwise, if $s^{\prime} \in \Lambda_{g, l}$ then by (19), there exists a $Q \in\left[q_{g}, Q_{g}\right]$ such that $s(Q)=s^{\prime}$. This leads to a contradiction to the assumption that $s(Q)$ is not continuous. Therefore, $s(Q)$ is continuous. See Figure 4 for an illustration of $s(Q)$.

Next we show that $s(Q)+Q$ is a nondecreasing function of $Q$. Instead of working on $s(Q)+Q$ directly, we define $S(Q)$ similarly to $s(Q)$, and show that $S(Q)=s(Q)+Q$. The definition of $S(Q)$ is

$$
S(Q)= \begin{cases}y^{*}+\frac{1}{2} Q & \text { if } Q \leq\left|\Lambda_{0}\right|, \\ S_{g(Q)}^{*} & \text { if } Q>\left|\Lambda_{0}\right| \text { and }\left|\Lambda_{g(Q), l}\right|=\left|\Lambda_{g(Q), r}\right|=0, \\ S_{g(Q)}^{*}+(1-p)\left(Q-q_{g(Q)}\right) & \text { otherwise. }\end{cases}
$$

We note that when $Q>\left|\Lambda_{0}\right|$, we have $\left|\Lambda_{g(Q), l}\right|=\left|\Lambda_{g(Q), r}\right|=0, S_{g(Q)}^{*} \in \Lambda_{g(Q), r}$, and $s_{g(Q)}^{*} \in \Lambda_{g(Q), l}$, so by the definition of $\Lambda_{g(Q), l}$ and $\Lambda_{g(Q), r}$, it follows that $S(Q)-s(Q)=Q$. Otherwise, when $Q>\left|\Lambda_{0}\right|,\left|\Lambda_{g(Q), l}\right| \neq 0$ or $\left|\Lambda_{g(Q), r}\right| \neq 0$, we have

$$
\begin{aligned}
S(Q)-s(Q) & =S_{g(Q)}^{*}+(1-p)\left(Q-q_{g(Q)}\right)-s_{g(Q)}^{*}+p\left(Q-q_{g(Q)}\right) \\
& =\left(S_{g(Q)}^{*}-s_{g(Q)}^{*}\right)+\left(Q-q_{g(Q)}\right) \\
& =q_{g(Q)}+Q-q_{g(Q)} \\
& =Q
\end{aligned}
$$

and lastly, when $Q \leq\left|\Lambda_{0}\right|, S(Q)=s(Q)+Q$ is easy to show. Hitherto, we have shown that $S(Q)=s(Q)+Q$. Thus, $S(Q)$ is continuous since $s(Q)$ is continuous.

The proof for $S(Q)$ to be nondecreasing is a counterpart of $s(Q)$ being nonincreasing, and it can be proven similarly.

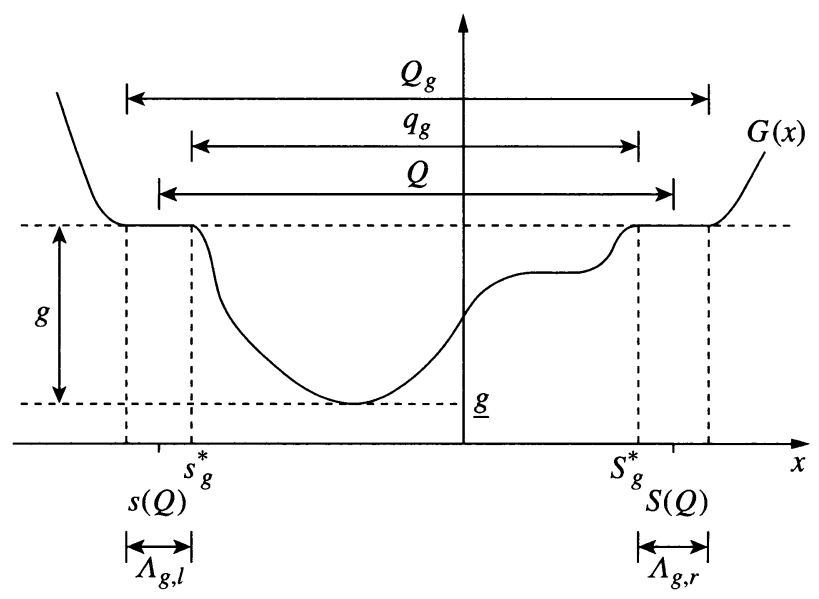

FIGURE 4: Illustration of $s(Q)$. 


\section{Acknowledgements}

The authors would like to thank an anonymous referee for the detailed and constructive comments and suggestions that have helped to significantly improve this paper. The research of Dacheng Yao is supported by the National Natural Science Foundation of China (grants 11401566 and 11101050). The research of Xiuli Chao and Jingchen Wu is supported in part by the National Science Foundation (grant nos CMMI-1131249 and CMMI-1362619).

\section{References}

[1] Bather, J. A. (1966). A continuous time inventory model. J. Appl. Prob. 3, 538-549.

[2] Constantinides, G. M. and Richard, S. F. (1978). Existence of optimal simple policies for discounted-cost inventory and cash management in continuous time. Operat. Res. 26, 620-636.

[3] DAI, J. G. AND YAO, D. (2013). Brownian inventory models with convex holding cost, Part 1: Average-optimal controls. Stoch. Systems 3, 442-499.

[4] DaI, J. G. AND YAO, D. (2013). Brownian inventory models with convex holding cost, Part 2: Discount-optimal controls. Stoch. Systems 3, 500-573.

[5] Dharmadhikari, S. and Joag-Dev, K. (1988). Unimodality, Convexity, and Applications. Academic Press, Boston, MA.

[6] HARrison, J. M. (1985). Brownian Motion and Stochastic Flow Systems. John Wiley, New York.

[7] Harrison, J. M. And Taylor, A. J. (1978). Optimal control of a Brownian storage system. Stoch. Process. Appl. 6, 179-194.

[8] Harrison, J. M., Sellke, T. M. ANd Taylor, A. J. (1983). Impulse control of Brownian motion. Math. Operat. Res. 8, 454-466.

[9] HaX, A. C. And CandeA, D. (1984). Production and Inventory Management. Prentice-Hall, Englewood Cliffs, NJ.

[10] Heyman, D. P. and Sobel, M. J. (2004). Stochastic Models in Operations Research, Vol. I, Stochastic Processes and Operating Characteristics. Dover, Mineola, NY.

[11] Ormeci, M., DaI, J. G. AND VANDE VATE, J. (2008). Impulse control of Brownian motion: the constrained average cost case. Operat. Res. 56, 618-629.

[12] Porteus, E. L. (1971). On the optimality of generalized $(s, S)$ policies. Manag. Sci. 17, 411-426.

[13] Porteus, E. L. (1972). On the optimality of generalized $(s, S)$ policies under uniform demand densities. Manag. Sci. 18, 644-646.

[14] RICHARD, S. F. (1977). Optimal impulse control of a diffusion process with both fixed and proportional costs of control. SIAM J. Control Optimization 15, 79-91.

[15] SCARF, H. (1960). The optimality of $(s, S)$ policies in the dynamic inventory problem. In Mathematical Methods in the Social Sciences, 1959, Stanford University Press, pp. 196-202.

[16] Sulem, A. (1986). A solvable one-dimensional model of a diffusion inventory system. Math. Operat. Res. 11, $125-133$.

[17] WU, J. AND ChaO, X. (2014). Optimal control of a Brownian production/inventory system with average cost criterion. Math. Operat. Res. 39, 163-189.

[18] ZHENG, Y.-S. (1992). On properties of stochastic inventory systems. Manag. Sci. 38, 87-103. 